\title{
Patient retention and satisfaction with information exchange concerning peripheral nerve block risks
}

\author{
Christopher M. Burkle, MD, JD • Catherine W. Njathi, MD • David E. Stoike, DO • \\ Christopher S. Johnson, MD • Allan M. Klompas, MB, BCh, BAO • Adam D. Niesen, MD • \\ Adam K. Jacob, MD
}

Received: 10 May 2017/Revised: 12 June 2017/ Accepted: 6 July 2017 / Published online: 12 July 2017

(C) Canadian Anesthesiologists' Society 2017

\section{To the Editor,}

The patient informed consent process facilitates "preference sensitive health care decisions" - described as a situation in which a patient may have more than one appropriate option. ${ }^{1,2}$ This description includes a patient's decision whether to proceed with a peripheral nerve block (PNB) to help manage postoperative pain. In one recent study from the United Kingdom, patients recalled the surgical risks of shoulder surgery better than the risks associated with their interscalene nerve block. ${ }^{3}$ Guidelines published earlier this year by the Association of Anaesthetists of Great Britain and Ireland suggest that information exchange about anesthesia risks occurs early and via leaflets or online resources. ${ }^{4}$

Patients undergoing orthopedic surgery at our institution are provided information on risks associated with PNB in written form several days or weeks in advance of their surgery and again verbally on the day of surgery. To determine the effectiveness of our existing PNB informed consent process, we assessed the level of retention orthopedic surgery patients had for the communicated risks. In addition, we determined patient satisfaction and modality preferences regarding PNB risk communication.

Following Mayo Clinic Institutional Review Board approval, 146 adult patients undergoing orthopedic surgical procedures with PNBs between July 15, 2015 and November 30, 2015 as well as between September 15, 2016 and November 15, 2016 were included in our study.

C. M. Burkle, MD, JD ( $₫) \cdot$ C. W. Njathi, MD

D. E. Stoike, DO - C. S. Johnson, MD - A. M. Klompas, MB,

BCh, BAO - A. D. Niesen, MD - A. K. Jacob, MD

Department of Anesthesiology and Perioperative Medicine,

Mayo Clinic, Rochester, MN, USA

e-mail: burkle.christopher@mayo.edu
Patients received a booklet during their preoperative evaluation clinic visit that provided information on the risks associated with their anesthetic care, including PNB risks. On the day of surgery (in the preoperative holding area), a discussion between the patient and the anesthesiologist took place that included information on risks associated with the application of PNB. On the day following surgery, patients were provided a written questionnaire asking them to indicate the PNB risks of which they were made aware preoperatively. The options on the questionnaire included three true risks (infection, nerve injury, bleeding), which were provided in both the informational pamphlet they received and during the conversation with their anesthesia provider on the day of surgery. Two distractors (nausea/vomiting, decreased effectiveness of other pain medications) were also included. Satisfaction with information delivery regarding PNB risks was also queried. In addition, we asked for their preferences for information exchange (timing, mode) regarding PNBs.

After surgery, patients could recall the following risks: infection $88.2 \%$, nerve damage $66.4 \%$, bleeding $66.9 \%$. Interestingly, $75.2 \%$ and $29.7 \%$ of patients recalled discussions about nausea/vomiting and decreased drug efficacy (distractors), respectively. Correct recall of risk factors and distractors is illustrated in the Figure. Overall, $71(48.6 \%)$ patients correctly listed all three of the true PNB risk factors. None of the patients correctly recalled all PNB risks and none of the distractors. There was no association of age, sex, or level of education on their ability to recall any of the specific risk factors or distractors. Although most patients preferred to receive information via personal discussion with the anesthesia provider alone (29.5\%) or in combination with an informational brochure $(63.0 \%)$, only $54.1 \%$ could recall receiving the 


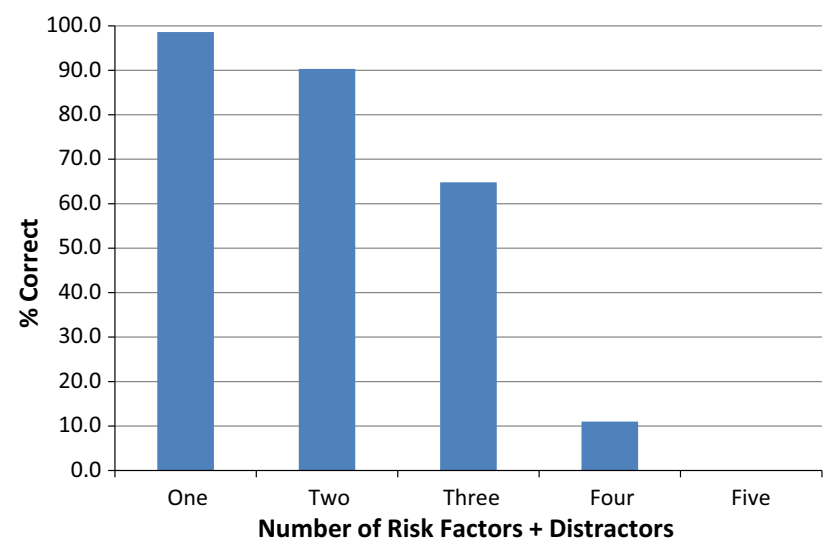

Figure Accurate recall of risk factors and distractors

informational booklet preoperatively. Overall, a majority of patients $(80.1 \%)$ also stated that they were very satisfied with the communication of PNB risks.

The poor patient recall of PNB risks points to a need to improve information exchange for orthopedic surgery patients at our institution. Although no apparent PNBrelated complications occurred in our study patients, poor risk recall could cause significant concern to an injured patient. Although patients reported high satisfaction with use of both written material and personal communication with their anesthesiologists, the hope is that a more reliable means of ensuring retention of PNB risks will lead to even greater appreciation of informed patient decision making.

Conflicts of interest None declared.

Editorial responsibility This submission was handled by Dr. Gregory L. Bryson, Deputy Editor-in-Chief, Canadian Journal of Anesthesia.

Financial sources This research did not receive any specific grant from funding agencies in public, commercial, or not-for-profit sectors.

\section{References}

1. Green DS, MacKenzie CR. Nuances of informed consent: the paradigm of regional anesthesia. HSS J 2007; 3: 115-8.

2. Veroff D, Marr A, Wennberg DE. Enhanced support for shared decision making reduced costs of care for patients with preferencesensitive conditions. Health Aff (Millwood) 2013; 32: 285-93.

3. Zarnegar R, Brown MR, Henley M, Tidman V, Pathmanathan A. Patient perceptions and recall of consent for regional anaesthesia compared with consent for surgery. J R Soc Med 2015; 108: 451-6.

4. Yentis SM, Hartle AJ, Barker IR, et al. AAGBI: Consent for anaesthesia 2017: Association of Anaesthetists of Great Britain and Ireland. Anaesthesia 2017; 72: 93-105. 